\title{
Nanoimprint lithography process chains for the fabrication of micro- and nanodevices
}

\author{
Helmut Schift \\ Laboratory for Micro- and Nanotechnology, Paul Scherrer Institut, 5232 Villigen PSI, Switzerland
}

\begin{abstract}
The nanoimprint lithography (NIL) process with its key elements molding and thin film pattern transfer refers to the established process chain of resist-based patterning of hard substrates. Typical processes for mass-fabrication are either fast wafer-like substrate imprint or to continuous foils by roll-to-roll processes. In contrast to this, similar process chains were established for polymeric micro-elements fabricated by injection molding, particularly when surface topographies need to be integrated into monolithic polymer elements. NIL needs to be embedded into the framework of general replication technologies, with sizes ranging from nanoscopic details to macroscopic entities. This contribution presents elements of a generalized replication process chain involving NIL and demonstrates its wide application by presenting a non-typical NIL product such as an injection molded micro-cantilever. Additionally a hybrid approach combining the NIL and injection molding in a single tool is presented. Its aim is to introduce the toolbox approach for nanoreplication into NIL-based processing and to facilitate the choice of suitable processes for micro- and nanodevices. By proposing a standardized process flow as described in the NaPANIL Library of Processes, the use of establish process sequences for new applications will be facilitated.
\end{abstract}

Keywords: Nanoimprint lithography, injection molding, microcantilever, micromechanical elements, polymer

\section{INTRODUCTION}

Molding processes leading to surface patterned polymer components consist of three principal processes: Origination, tooling and replication, often followed by post-processing steps such as assembly, integration into frames with a macroscopic interface and packaging. This is valid for replication processes for components with different structure sizes and applications, such as surface topographies for diffractive optical elements (DOE) ${ }^{1-4}$ up to high aspect ratio microstructures for micromechanical elements with microfluidic channels ${ }^{5-9}$. These principal processes involve many more process steps, i.e. design, process simulation, stamp copying, transformation into a working tool with appropriate structural resolution, area enlargement and even the integration of mix micro- and nanostructures with three-dimensional (3D) features. When combined to a sequence, it is called the process chain. For production, design issues are closely interlinked with each process step and determine, whether the goals of the end-user can be met. This results in a value chain where not only technological aspects, but also cost of ownership needs to be considered, i.e. costs of equipment, backup, infrastructure and manpower. Where standard processes are difficult to establish, processes need to be selected from a process pool ranging from established microfabrication processes to approaches and materials used in research. This is called replication toolbox. In the following, two main processes using this toolbox are presented.

\section{NANOIMPRINT LITHOGRAPHY}

Nanoimprint lithography (NIL) relies on the same toolbox but with two main differences ${ }^{10-13}$ : It uses silicon micromachining techniques for stamp fabrication but also as substrates. And it heads towards thin polymeric films where the sizes and heights of the structures become comparable to the films to be patterned. Due to its large potential as a manufacturing process for nanoscale surface topographies, its definition as "lithography" is only valid for specific applications. The process chain is therefore often composed of origination, replication and pattern transfer, in which the last step is the transformation of the surface topography in the thin polymer film into a different material, e.g. by using it as an etch mask for the substrate or for metallization. This process chain is depicted in Figure 1.

*helmut.schift@psi.ch; phone ++41-56-310-2839; fax ++41-56-310-2646; www.psi.ch

Alternative Lithographic Technologies VI, edited by Douglas J. Resnick, Christopher Bencher, Proc. of SPIE Vol. 9049, 90491E · C 2014 SPIE · CCC code: 0277-786X/14/\$18 · doi: 10.1117/12.2048517 


\section{Origination (stamp/mold fabrication and tooling)}

$\begin{aligned} & \text { Electron beam } \\ & \text { lithography }\end{aligned}$
$\begin{aligned} & \text { Wet resist } \\ & \text { development }\end{aligned}$

\section{Replication (thermal, UV-, step\&repeat and roll-to-roll NIL)}

Stamp alignment
on resist coated
substrate
spin-coated film
or dispensed droplets
Thermal or
UV-light assisted
nanoimprint
viscous squeeze and
capillary induced flow

Demolding
stamp detachment
from molded resist
(thickness contrast)
and re-use
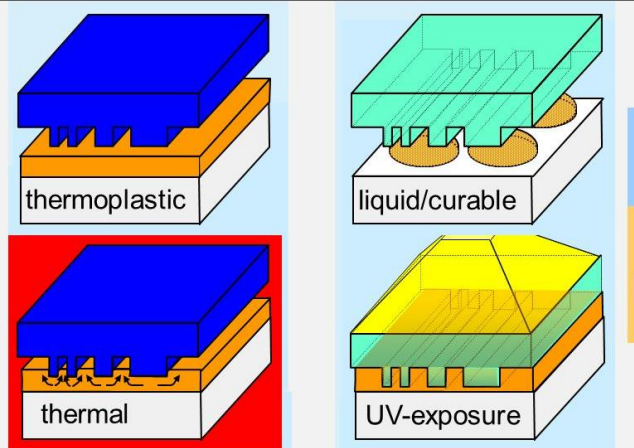

Lateral area enhancement by multiple imprint (step\&repeat)

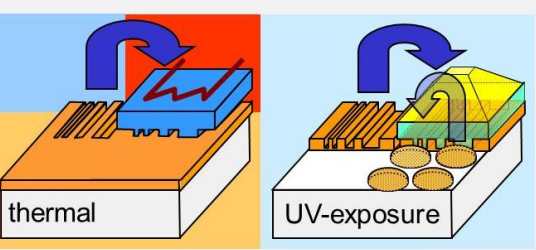

Roller imprint (dynamic/continuous) roll-to-role and roll-to-plate
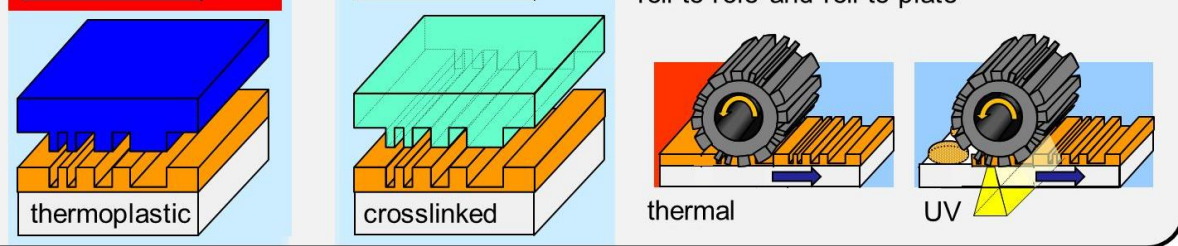

\section{Pattern transfer (window opening and substrate patterning)}

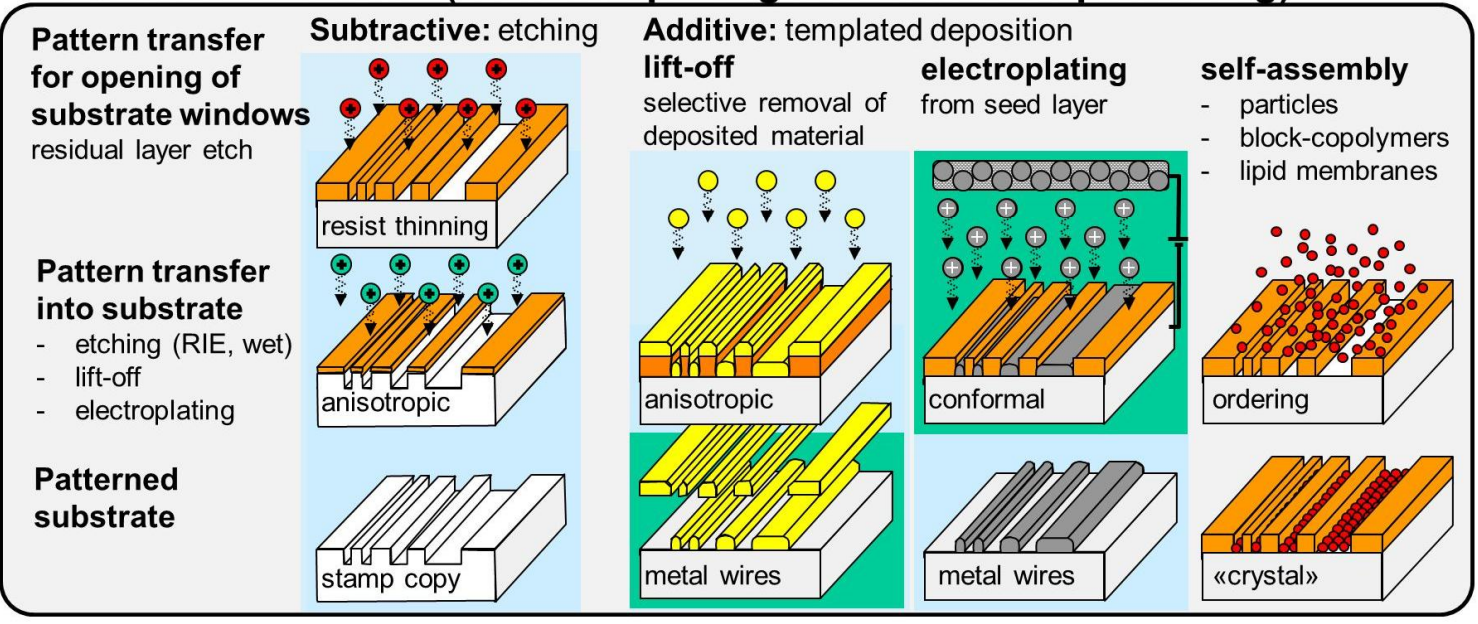

Figure 1. Nanoimprint lithography consisting of the three major process steps origination, replication and pattern transfer. 
The existence of many choices of processes and variants thereof, with a high interdependence of steps, makes it difficult to select them for a process chain without knowing their requirements from previous and consequences for following steps in the chain. In Figure 1, the left column of processes depicts the standard NIL process (with its main replication variants thermal and UV-assisted NIL). While stamp copying and tooling are added to the origination, step\&repeat and roller NIL variants are added to the replication section. Particularly interesting are resolution enhancement methods by using spacer etching techniques and directed self-assembly of block copolymers ${ }^{14-15}$. In pattern transfer, only the most prominent processes are displayed. In Figure 2, the resulting process chain is presented.

Because mold manufacturing needs the knowledge base of specialists coming from other disciplines than toolmakers, mold origination uses a range of processes with specific restrictions in design, sizes and material. The original sometimes may be directly used for replication, but often does not yet fulfill the requirements of the molding process in terms of size, flexibility or durability. Therefore a replication process is used for generation of single, or even multiple mold copies. For several reasons, metal molds are preferred by industrial customers, and electroplating has become the process making it possible to copy the surface topography of an original into a tool which meets the boundary process of high throughput manufacturing for processes outside a cleanroom. For manufacturing, the original with the surface topography has therefore to be transformed into a working tool adapted to the molding process, which means that a suitable mold outline has to be generated, which can be integrated into the tool. The original polarity is inverted during the copying process. Once such a mold is generated, it can be repeatedly used to generate identical copies without intentional degradation of the mold. Special tooling efforts are needed if the original needs to be transformed or copied into different material, to be enlarged in area or simply to be fitted into a standardized tool.

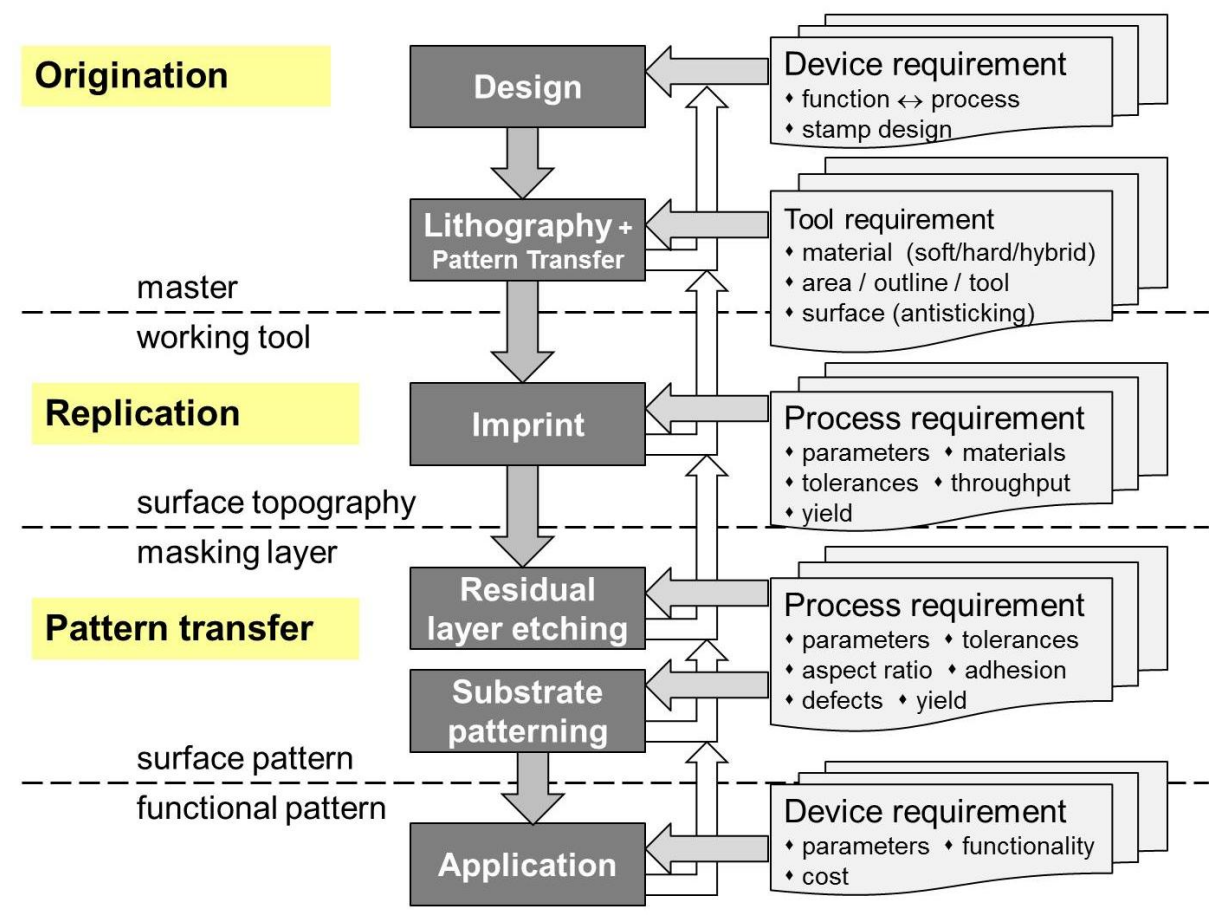

Figure 2. Nanoimprint process chain including feedback loops for process and device requirements .

Replication techniques are manifold, most prominent are casting, imprint (embossing) and injection molding. The basic process is shaping of a material in its viscous shape by redistribution and hardening before the mold is separated (demolding). In most cases the final element exhibits the exact shape of the mold outlines including surface patterns, however with a "negative" tone, as already mentioned for mold copying. Depending on the viscosity of the molded material, the viscous material wets and covers the mold surface and the voids by pressure or capillary driven filling. Either the mold is designed hard enough that it can be reused without damage, or soft and flexible enough that it allows being detached without wear. This is often depending on the size and high aspect ratio. While molding processes dealing with surface structures with moderate aspect ratio structures often rely on flat stamper and roll-to-roll embossing, injection molding enables to mold entire components with defined outlines. This is used for a range or polymeric 
products such as compact discs (CD). Here, a thin metal stamper is electroplated from a resist coated glass substrate and inserted into an injection molding tool. Thus the sub-micrometer data pits, which have been patterned into the resist by a focused laser beam, are replicated onto the surface of a polymer disc with $120 \mathrm{~mm}$ diameter. Similar results can be achieved with much smaller structures, e.g. for Bluray discs. Apart from these standard processes, in a research project silicon wafers were used instead of electroplated stamps and down to $25 \mathrm{~nm}$ resolution was achieved ${ }^{16-19}$. Also stamps with surface structures in hybrid organic-inorganic polymers (e.g. Ormostamp ${ }^{\circledR}$ from micro resist technology GmbH, Berlin), were used and sub-100 nm patterning demonstrated ${ }^{20-21}$.

Pattern transfer is often not needed if the end-product is made from polymer or, as in case of NIL, a stamp copy is fabricated by replication ${ }^{20}$. However, pattern transfer makes it possible to use the polymer pattern as a masking layer and transform its lateral design it into a different material. In practice this is used in manufacturing of microchips for the lithography of single or multiple layers. For polymeric elements, also thin film generation and processes are essential to generate additional properties, e.g. such as the enhancement of reflectivity. For CDs, as presented before, a metal coating has to be integrated in the process chain to enable the readout by a focused laser. In the case of the CDs, this would be readout of single information pits with a focused laser with about $1 \mu \mathrm{m}$ beam size. Many more products of our daily life rely on molding processes, and use polymer elements with an additional coating. Two further examples are patterned magnetic media where entire discs have to be patterned before a magnetic film is added, or thin holographic security labels which cover bank notes and credit cards. In all these processes, replication is one essential process to replicate an original surface pattern. Process chains have been developed for a range of other replication products, e.g., micro-optical components in smartphones. Although the processes for origination, replication and pattern transfer often look quite different, their ingredients often come from the same family of processes. In the following, this is exemplified on the basis of a polymeric microcantilever $(\mu \mathrm{C})$ which uses the process chain of the $\mathrm{CD}$ molding. By adding surface patterns to these $\mu \mathrm{Cs}$, injection molding is combined with NIL surface patterning capabilities.

\section{POLYMER MICROCANTILEVERSPROCESS CHAIN LIBRARY CONCEPT}

A microcantilever $(\mu \mathrm{C})$ is a micromechanical device which exhibits flexible, fiber-like extensions from a macroscopic carrier which can bend if they are submitted to surface stress or excited by mechanical forces. This can e.g. be by loading a $\mu \mathrm{C}$ with biomolecules which will result in a decrease of its resonance frequency. To achieve local chemical sensitivity, the $\mu \mathrm{C}$ is coated on one side with a thin gold-film. This serves both as a layer for selective adsorption of biomolecules (e.g. by using thiol-based chemistry) and for enhancing the reflection of a laser beam, which is used to measuring the deflection of the beam upon. Typically, a $\mu \mathrm{C}$ is $500 \mu \mathrm{m}$ long, 50 to $100 \mu \mathrm{m}$ wide and around $1 \mu \mathrm{m}$ thick. As a typical device with multiple sensors, an array of $\mu \mathrm{Cs}$ is attached to a $2.5 \times 3.5 \times 0.5 \mathrm{~mm}^{3}$ carrier. While the size of the carrier is determined by practical considerations (handling with tweezers, economy of space and thickness of standard silicon wafers), the size of the $\mu \mathrm{Cs}$ is often determined by the selectivity needed to detect small amounts of loaded biomolecules and variations thereof. Silicon is the preferred material for these micromechanical elements, due to the established capabilities of cleanroom-based micromachining technologies. However, particularly for sensors in a modern bio-lab environment, single-use low-cost devices are highly desired. For this, polymeric alternatives are most promising. The preferred manufacturing process for those $\mu$ Cs is polymer injection molding, and for micro-elements of a few millimeter size special micro injection molding $(\mu \mathrm{IM})$ tools and concepts for have been developed. To achieve comparable mechanical properties of polymeric $\mu \mathrm{C}$, the polymer $\mu \mathrm{Cs}$ need to be around 7 times thicker than their silicon counterparts. However, even a cavity height of $10 \mu \mathrm{m}$, i.e. with an aspect ratio of 50, is difficult to fill with a polymer melt in an isothermal process, i.e. in which the mold is kept at a temperature below the melt's glass transition temperature. Here, the thermoplastic polymer needs to keep its ability to flow and fill the extended cavity, while it is freezing upon contact with the mold surface. However, as has been demonstrated, complete filling can be achieved if the mold cavity height is chosen between 30 to $50 \mu \mathrm{m}$. This is also possible if one mold surface is covered with micro- and nanostructures. From a process point of view, the shaping of an entire CD with $120 \mathrm{~mm}$ diameter and $1.2 \mathrm{~mm}$ thickness (DVD $0.6 \mathrm{~mm}$ ) or a millimeter sized polymer holder with some $500 \mu \mathrm{m}$ long and $25 \mu \mathrm{m}$ thick fiber-like extensions is not different. Even for $25 \mu \mathrm{m}$ thick $\mu \mathrm{Cs}$, their aspect ratio is much higher than 10 and rarely achieved in NIL processes. This is also true if the $\mu \mathrm{Cs}$ are patterned on their surface with sub- $\mu \mathrm{m}$ sized holes or pillars. Since the surface patterning is achieved by using a NIL fabricated hybrid mold, the NIL toolbox approach is needed. A process for this is schematically depicted in Figure 3. Opposite to the microcavity with the $\mu \mathrm{C}$ outlines on the tool side, a foil containing the surface pattern is attached to the injection (mirror) side of the mold. Thus, by composing a hybrid mold cavity during closing of the tool, instead of the flat surface, a surface pattern is generated on the $\mu \mathrm{C}$ during molding. 


\section{Origination (mold inserts and inlays)}

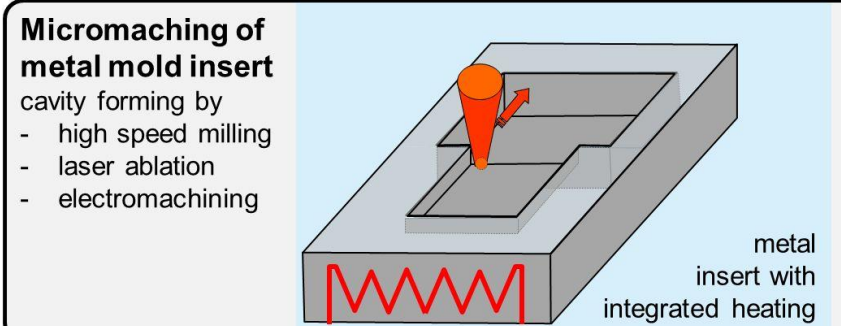

\section{Patterning of}

polymer foil mold replication of stamp surface topography by

- thermal imprint

- casting and curing electroplating integrated heating

\section{Replication (of surface patterned microcantilevers)}

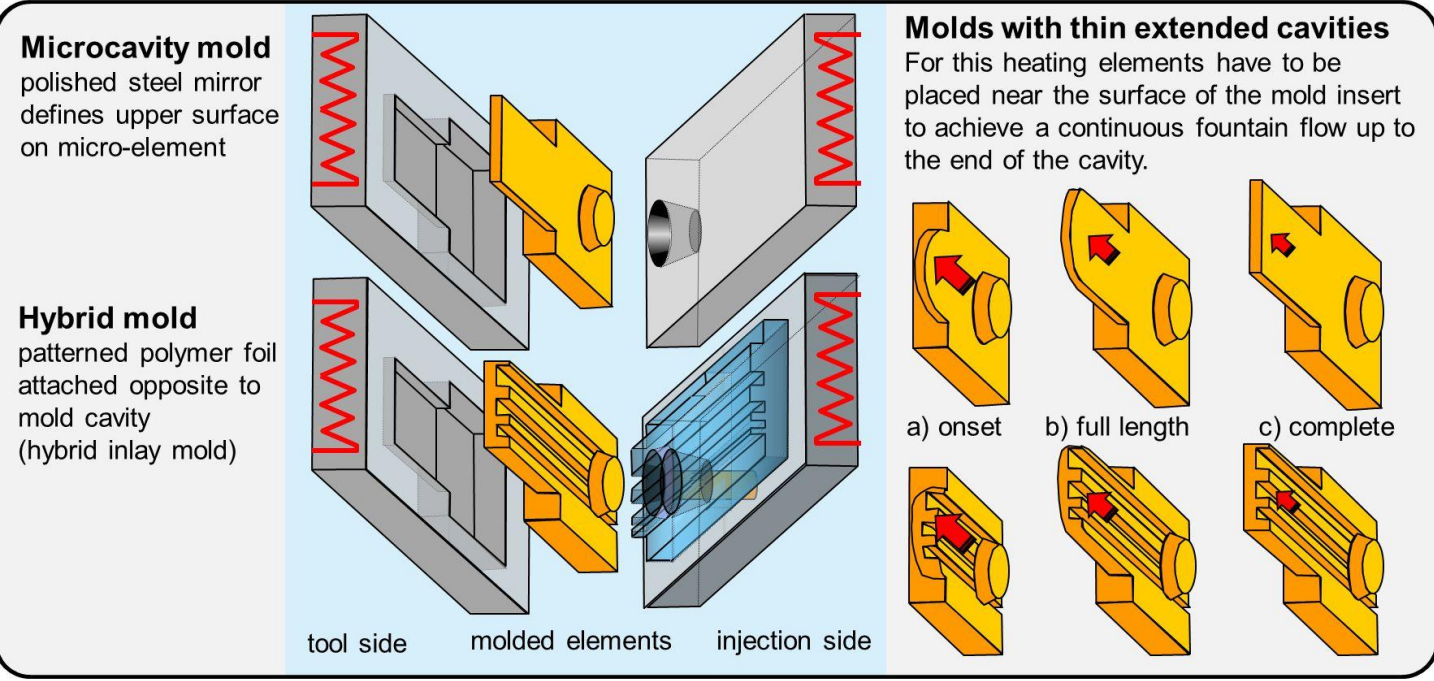

\section{Injection cycle (mold filling and ejection)}

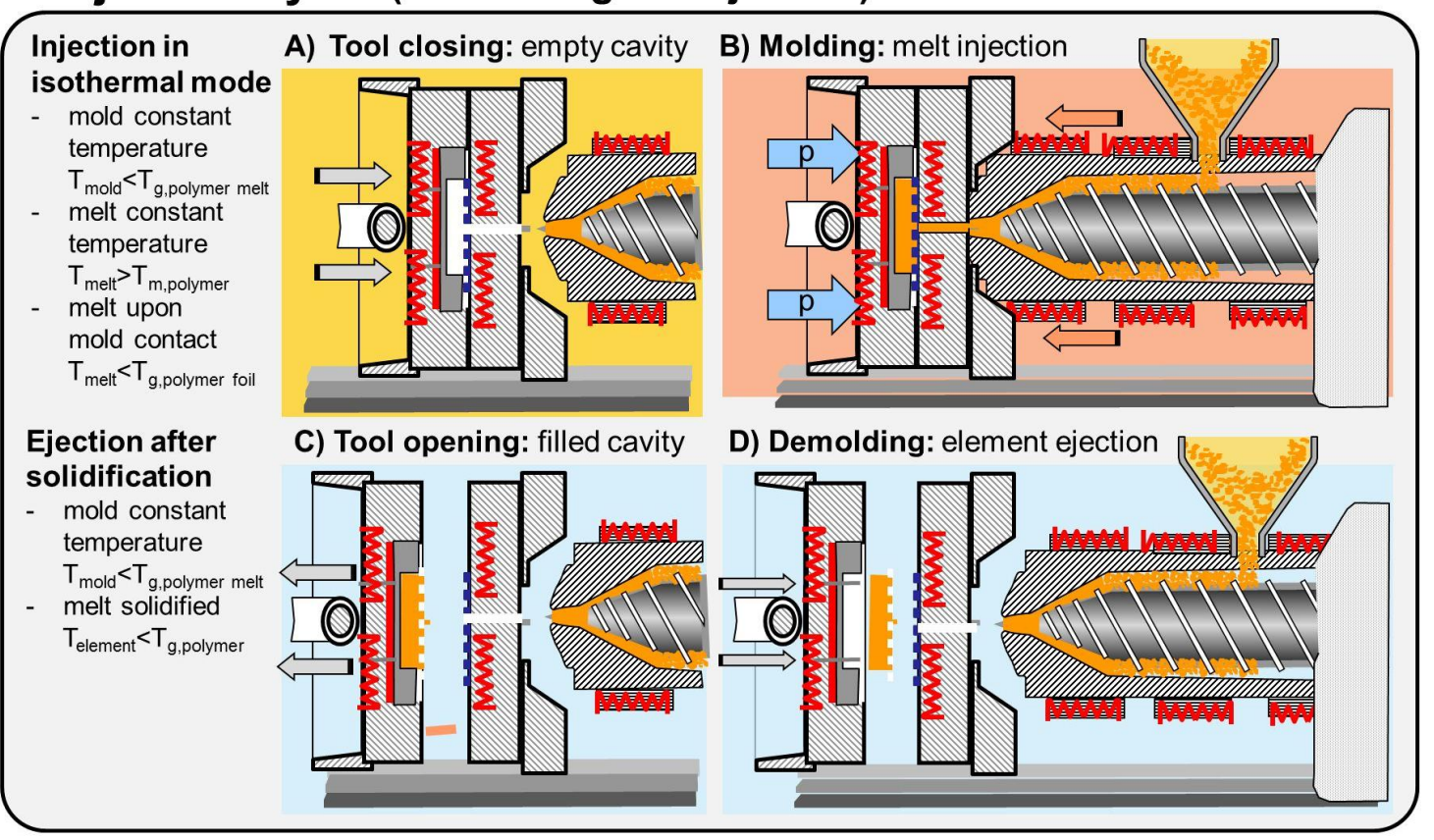

Figure 3. Process for the fabrication of a surface patterned microcantilever device by micro injection molding. 
Most of the process details have already been presented. In Ref. 22 and 23 the fabrication of the polymer $\mu$ Cs was described, including the integration of surface corrugations by hybrid molds. This concept has also similarities with the so-called "in-mold labelling" of consumer goods, were a foil is integrated into a mold and permanently reinforced by the injected polymer. Thus a printed film can be used to decorate products, yielding high wear-resistance. By interchanging the foil, different surface patterns can be applied without changing the entire mold. In this process, NIL is only used for the "hybrid part" of the replication, resulting in a "decoration" of the $\mu \mathrm{C}$, without an impact on their mechanical properties. However, the surface area is changed, e.g. doubled if gratings with of an aspect ratio of 1 (e.g. lines and spaces of $1 \mu \mathrm{m}$ each, depth $1 \mu \mathrm{m}$ ), and may enhance the sensitivity of the $\mu \mathrm{C}$ 's surface. Alternatively, depending on the depth of the micro-gratings with respect to the $\mu \mathrm{C}$ 's thickness, the mechanical properties can be modified, too, e.g. depending on the orientation of the gratings the cantilever can be made stiffer or softer. However, this application is meant to demonstrate that there are possibilities to add functionality to a microelement by modifying its surface.

In Figure 4 the process chain for $\mu \mathrm{C}$ manufacturing by $\mu \mathrm{IM}$ is depicted. As seen for the NIL process in Figure 2, its main task for origination is the fabrication of the two mold parts. The microcavity of the $\mu \mathrm{C}$ array is milled into a prefabricated tool insert made from tool steel. Interestingly, while each microcavity is an original, the patterned foil has to be fabricated using a replication process. Therefore different original molds can be used and polymer backups can be provided. Furthermore, instead of the pattern transfer, the micromechanical device has to undergo a post-treatment, by cleaning it from organic residues and coating it with a thin gold layer ${ }^{24-26}$. Both processes modify the mechanical and even chemical characteristics of each $\mu \mathrm{C}$. Once this functionalization is done, the device is finished and can be used as a sensor device, e.g. in the Cantisens ${ }^{\circledR}$ cantilever sensor platform by Concentris GmbH in Basel, Switzerland ${ }^{27}$.

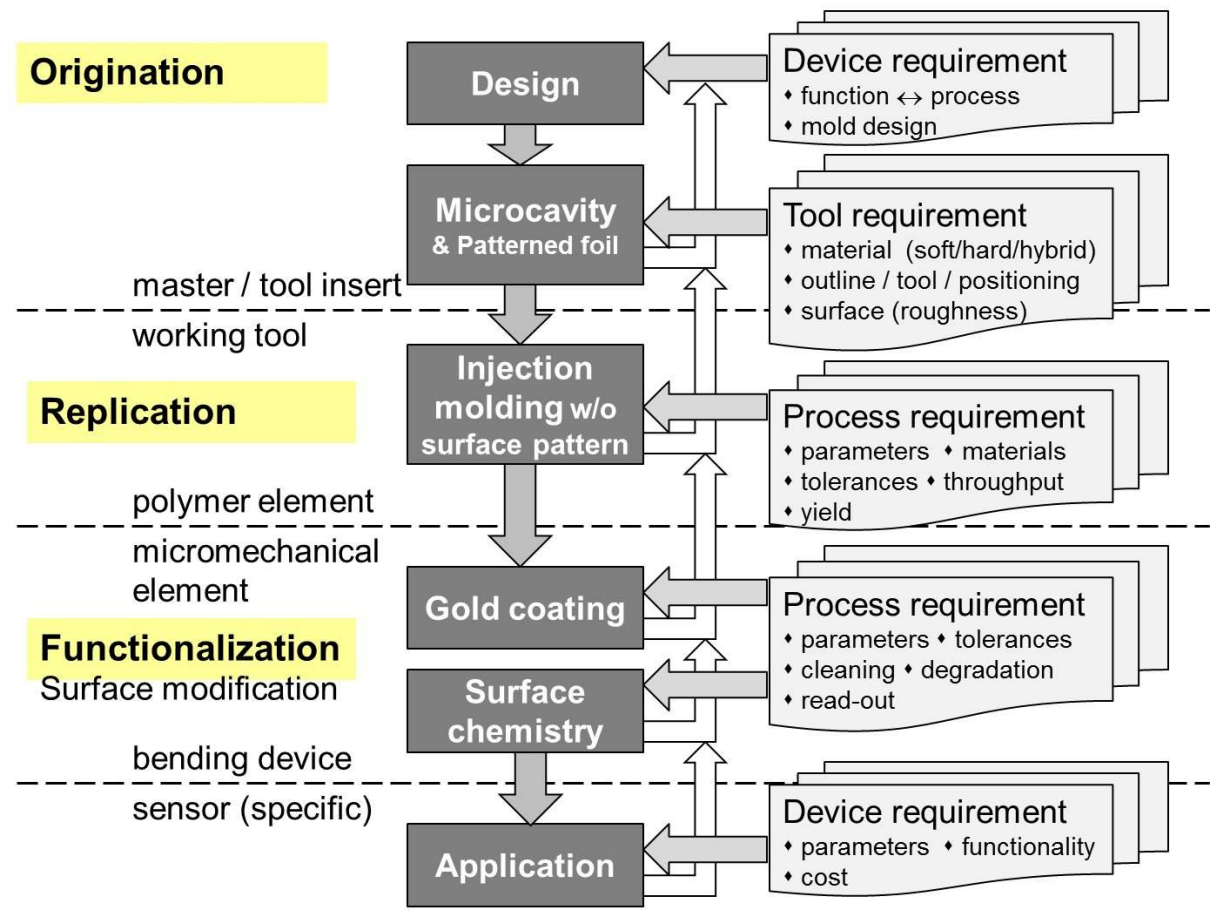

Figure 4. Microcantilever process chain including feedback loops for process and device requirements. In contrast to the NIL process chain, pattern transfer is replaced by surface functionalization.

Devices with thin membrane-like elements have been fabricated with a range of other methods, which would also be suitable for $\mu \mathrm{Cs}$, e.g. by micro-embossing of thin foils, by photolithography of thick resists or even by NIL. In all cases, single processes or entire process chains from differ from the fabrication of $\mu \mathrm{Cs}$. By using this, free-standing membranes with $1 \mu \mathrm{m}$ thickness were fabricated as sieves or as photonic crystal slabs ${ }^{28}$. 


\section{PROCESS CHAIN LIBRARY CONCEPT}

Many scientific publications, particularly those relying on state-of-the-art manufacturing techniques, present process details for the processing of devices with which research results were obtained. Although scientific publication requires to proof and justify results, with the aim to make it possible for others to verify the results, processes can be rarely considered as consolidated. The lack of standardization in research is commonly acceptable for those who work on similar issues, follow developments and are able to find own solutions which profit from the published details but are often not a direct copy of the processes presented. The need for standard processes is, however, not only a wish to facilitate the setup of new processes, but also a need to extract relevant information about single processes and their mutual interdependence in a process chain. This is particularly important if the suitability for scale-up and transfer into a real product has to be judged. A process chain description, even if not yet defined as a standard, can be valuable help to understand processes, compare results based on own processing knowledge. It can simply be a way to find and learn from processes which may be described in publications, but are not presented in a useful structure and logic.

Figure 5 presents four pages (a-d) of a process chain for the fabrication of surface patterned polymeric microcantilevers $(\mu \mathrm{C})$ from the NaPa library of processes $(\mathrm{NaPa} \mathrm{LoP})^{29}$. This LoPs are is the result of the European Integrated Project $\mathrm{NaPa}$ (2004-2008) and the Large Scale Project NaPANIL (2008-2012), which during a total of 8 years period gathered scientists and engineers to develop a range of nanopatterning method.

NaPANIL_Library of Processes

\subsection{Polymeric microcantilevers}

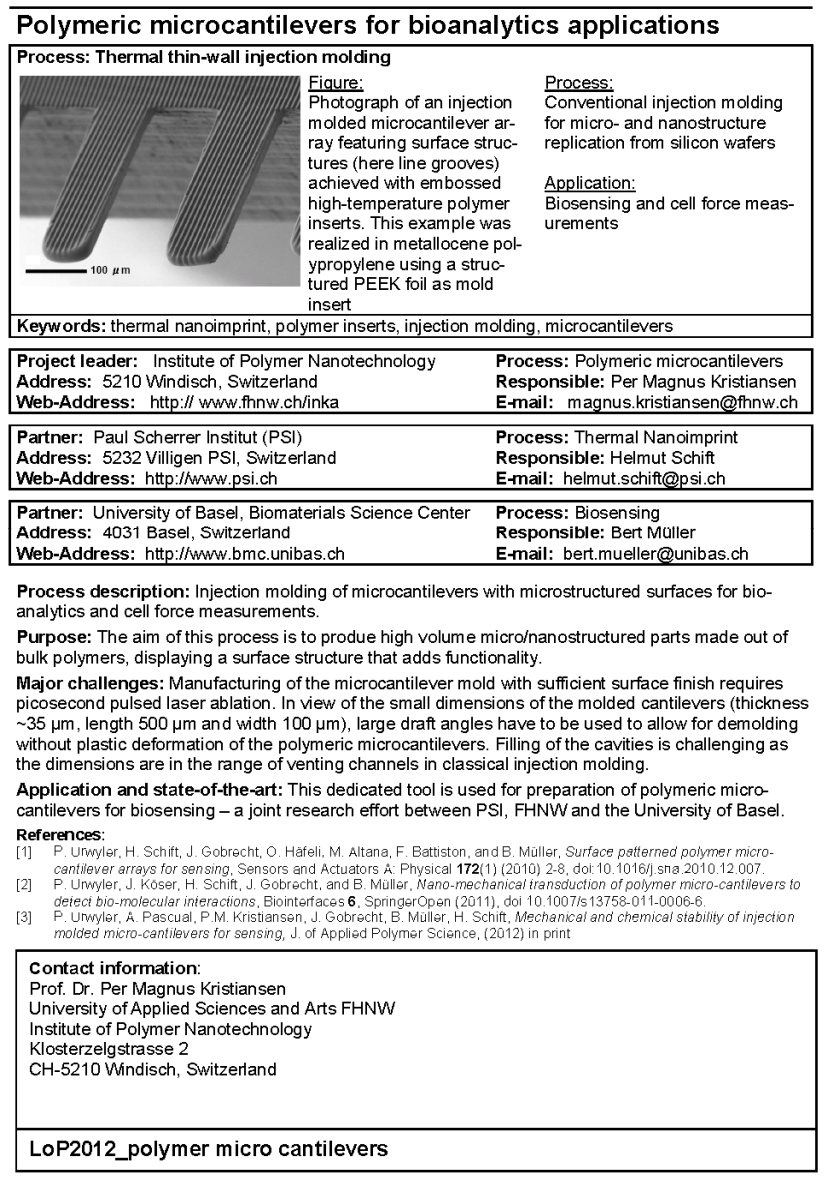

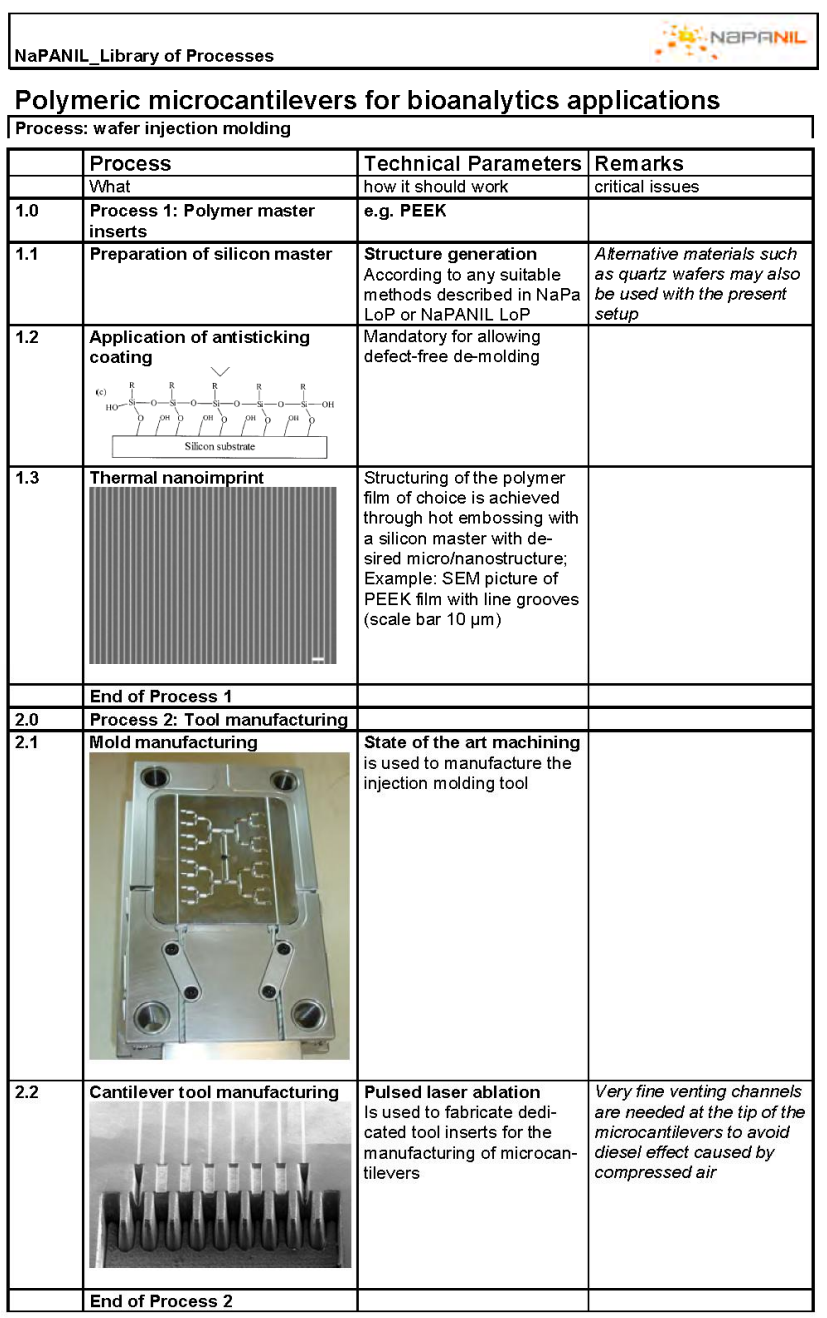

Figures 5a and b. Process for manufacturing of polymeric microcantilevers, reproduced from the NaPANIL Library of Processes $^{29}$, pages $199-200$, as an example for a process which enables user to identify process steps. The process was performed together with the University of Applied Sciences Northwestern Switzerland (FHNW). 

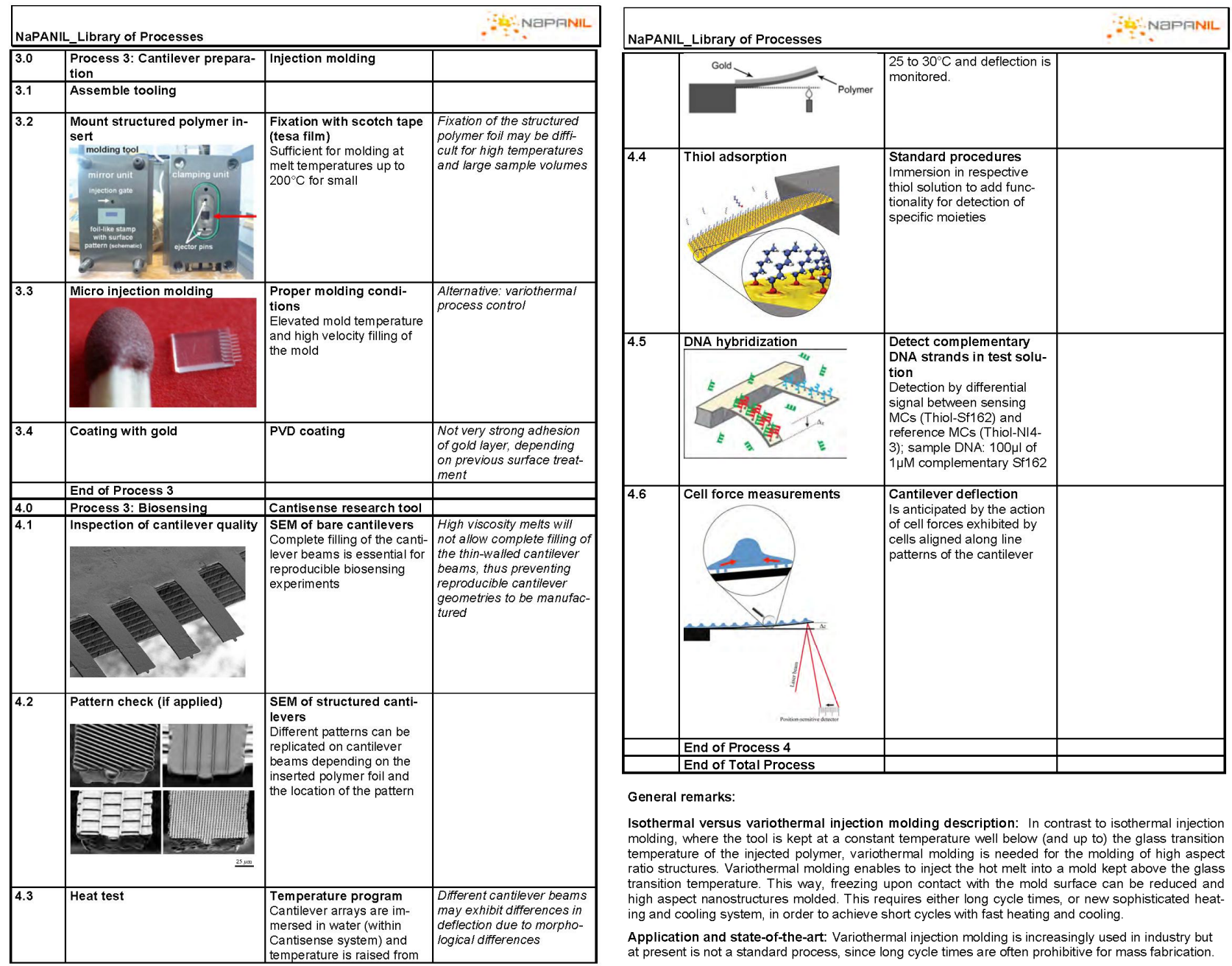

Figure $5 \mathrm{c}$ and $\mathrm{d}$. Process for manufacturing of polymeric cantilevers, reproduced from the the NaPa Library of Processes ${ }^{29}$, pages 201-202.

The library concept is not new, but it has been proved to be a valuable tool for documentation and dissemination. The benefit from bachelor, master or $\mathrm{PhD}$ works within an academic environment is often lost if this knowledge is not translated into a form which can be read by the technologically experienced researcher or engineer. Its main aim was therefore the leverage of technology take-up, particularly by small or medium sized enterprises (SME), which rarely follow the technological progress in scientific publications. These documents do not necessarily need to disclose confidential information or go beyond the range of details already presented in the publication, but help to structure the process and enable to assess the state-of-the-art. It gives information about both the toolbox and the process chain. It may contain work in progress, since many process variations were needed to achieve intermediate or the final results. However, even if a standard process is not yet established, the description of a semi-standard will allow learning process routes and the logic of achievements. For this, next the column giving technical parameters, remarks should be added. They are essential to put single steps or preliminary results into context. Apart from the fact, that a copying of the exact process is often not possible or wished, the starting at a point zero $+\Delta$ will allow to enhance the learning curve.

The NaPa LoP with more examples for NIL based microfabrication can be downloaded from the author's webpage at Paul Scherrer Institut ${ }^{29}$, and templates can be easily composed according to the example described above. The results are all coming from project partners of the NaPa and NaPANIL, and are, as a whole, have already proven to be a valuable resource for students and engineers starting with microfabrication processing. It is our aim that researchers and engineers take up this idea to present their processes in a similar format in web-based annexes of their publications. 


\section{CONCLUSION}

Many process chains for micro- and nanofabrication of devices have been established in research environments and are particularly important in industry for process control and documentation. The aim here is directed towards technology oriented research projects, and does not cover the cost-of-ownership oriented value chains guided by technological roadmaps. However, particularly because both NIL and $\mu \mathrm{IM}$ are replication techniques with the inherent potential for large scale manufacturing, the viability of process routes for scale-up needs to be addressed. This can be described by different technology readiness levels (TRL) ${ }^{30}$. TRL is a measure used to assess the maturity of evolving technologies (devices, materials, components, software, work processes, etc.) during its development and in some cases during early operations. In research, single elements of the chain are often labelled with different maturity levels, and highly dependent on structural designs and complexity. The aim of this publication is not to define these TRLs for the process chain presented, but to help establishing these process chains for future developments. The NIL process chain with its main elements origination, replication and pattern transfer (functionalization), is a good example to demonstrate this capability.

\section{ACKNOWLEDGEMENT}

The basis for writing this review on process chains for nanoimprint and related molding processes was the development of thermal NIL over the last 15 years in the Paul Scherrer Institut. Many thanks are due to all those researchers, engineers, technicians, and students, who contributed to the continuous development of the NIL technology in the Laboratory for Micro- and Nanotechnology (LMN) at PSI, and particularly K. Vogelsang, S. Park, M. Altana, C. Spreu and A. Schleunitz. The work on the microcantilevers was done by P. Urwyler-Harischandra, who is now affiliated with ARTORG Center for Biomedical Engineering Research in Bern, Switzerland. Her work was performed within the framework of INKA (Institute of Polymer Nanotechnology), a joint institute of the University of Applied Sciences and Arts Nordwestschweiz and the PSI. For the production of the NaPa library of processes (NaPa LoP) I would like to express my gratitude to all scientists and engineers, who participated in NaPa and NaPANIL and contributed to the LoP.

\section{REFERENCES}

[1] Gale, M.T., [Replication], in Micro-optics, H.P. Herzig, ed., Taylor \& Francis, London, 153-178 (1997).

[2] Gale, M.T., Rossi, M., Schuetz, H., "Fabrication of continuous-relief micro-optical elements by direct laser writing in photoresist, laser-assisted fabrication of thin films and microstructures," Proc. SPIE 2045, 54-62 (1994)

[3] Gale, M.T., "Replication techniques for diffractive optical elements," Microelectron. Eng. 34 (1997) 321-339

[4] Stäger, B., Gale, M.T. and Rossi, M., "Replicated micro-optics for automotive applications," Photonics in the Automobile, edited by Thomas P. Pearsall, Proc. SPIE 5663, 238-245 (2005).

[5] Becker E.W., Ehrfeld W., Hagmann P., Maner A., Münchmeyer D., "Fabrication of microstructures with high aspect ratios and great structural heights by synchrotron radiation lithography, galvanoforming, and plastic moulding (LIGA process)," Microelectron. Eng. 4(1), 35-56 (1986).

[6] Menz, W., Mohr, J., Paul, O., Mikrosystemtechnik für Ingenieure, 3rd edn. Wiley-VCH, Weinheim (2005).

[7] Saile, V., Wallrabe, U., Tabata, O., [LIGA and its Applications], in Advanced Micro and Nanosystems (Vol. 7), series eds. J.G. Korvink, O. Brand, G.K. Fedder, C. Hierold, 1st edn. Wiley-VCH, Weinheim (2008).

[8] Heckele, M., Schomburg W.K., "Review on micro molding of thermoplastic polymers," J. Micromech. Microeng., 14, R1 (2004).

[9] Worgull, M., Hot embossing, 1st edn. William Andrew, Oxford, UK (2009).

[10] Chou, S.Y., Krauss, P.R., Renstrom P.J., "Imprint of sub-25 nm vias and trenches in polymers," Appl. Phys. Lett. 67(21), 3114-3116 (1995).

[11] Schift, H. and Kristensen A., [Nanoimprint lithography - patterning resists using molding]. Chapter (Part A/9) in "Handbook of nanotechnology," Vol. ed. B. Bhushan, Springer Verlag Berlin Heidelberg, Germany, 271-312 (2010). 
[12] Schift, H., "Nanoimprint lithography: an old story in modern times? A review," J. Vac. Sci. Technol. B 26(2), 458-480 (2008).

[13] Schift, H., "Nanoimprint lithography and micro-embossing in LiGA technology: similarities and differences," Microsyst. Technol. (2013).

[14] Greschner, J. and Trumpp, H.-J., "Method of making structures with dimensions in the sub-micrometer range," US-patent US4502914-A (1985-03-05). International Business Machines Corporation. Priority: US 06/546,612, 1982-11-13.

[15] Ruiz R., Kang H., Detcheverry F.A., Dobisz E., Kercher D.S., Albrecht T.R., de Pablo J.J. and Nealey P.F., "Density Multiplication and improved lithography by directed block copolymer assembly," Science 321, 936939 (2008).

[16] Schift, H., David, C., Gabriel, M., Gobrecht, J., Heyderman, L.J., Kaiser, W., Köppel, S. and Scandella, L., "Nanoreplication in polymers using hot embossing and injection molding," Microelectron. Eng. 53, 171-174 (2000).

[17] Schift, H., D’Amore, A., David, C., Gabriel, M., Gobrecht, J., Kaiser, W. and Simoneta, D., "Quantitative analysis of the molding of nanostructures," J. Vac. Sci. Technol. B 18 (6), 3564-3568 (2000).

[18] Gobrecht, J., Schift, H., David, C., Kaiser, W., D’Amore, A., Simoneta, D. and Scandella, L., "Injection molded plastic chip for calibration of scanning probe microscopes," PTB Berichte PTB-F-39 Braunschweig, (eds. K. Hasche, W. Mirandé and G. Wilkening), Germany (2000), Proc. 4th Seminar on Quantitative Microscopy QM'2000, 1-7 (2000).

[19] D'Amore, A., Gabriel, M., Haese, W., Schift, H. and Kaiser, W., "Nanoreplikation - Informationsverdichtung," Kunststoffe 94, 54-58(2) (2004). English version: "Nano-replication - concentration of information," Kunststoffe plast europe 94(2), 4-7 (2004).

[20] Schift, H., Spreu, C., Saidani, M., Bednarzik, M., Gobrecht, J.. Klukowska, A., Reuther, F., Gruetzner, G. and Solak, H.H., "Transparent hybrid polymer stamp copies with sub-50 nm resolution for thermal and UVnanoimprint lithography,” J. Vac. Sci. Technol. B 27(6), 2846-2849 (2009).

[21] Schleunitz, A., Spreu, C., Mäkelä, T., Haatainen, T., Klukowska, A. and Schift, H., "Hybrid working stamps for high speed roll-to-roll nanoreplication with molded sol-gel relief on a metal backbone,", Microelectron. Eng. 88(8), 2113-2116 (2011).

[22] Urwyler, P., Häfeli, O., Schift, H., Gobrecht, J., Battiston, F. and Müller, B., "Disposable polymeric microcantilever arrays for sensing," Procedia Eng. 5, 347-350 (2010).

[23] Urwyler, P., Schift, H., Gobrecht, J., Häfeli, O., Altana, M., Battiston, F. and Müller, B., "Surface patterned polymer micro-cantilever arrays for sensing," Sensors and Actuators A: Physical 172(1), 2-8 (2011).

[24] Schift, H., Urwyler, P. and Kristiansen, P.M., "Surface-patterned micromechanical elements by polymer injection molding with hybrid molds," J. Vac. Sci. Technol. B 31(6), 06FD01 (8 pp.) (2013).

[25] Urwyler, P., Pascual, A., Kristiansen, P.M., Gobrecht, J., Müller, B. and Schift, H., "Mechanical and chemical stability of injection molded micro-cantilevers for sensing," J. Appl. Polym. Sci. 127(4), 2363-2370 (2013).

[26] Urwyler, P., Pascual, A., Gobrecht, J., Schift, H., and Müller, B., "Chemical stability of ultraviolet-ozone treated, injection-moulded poly lactic acid micro-cantilevers," European Cells and Materials 23, 36 (2012).

[27] Urwyler, P., Köser, J., Schift, H., Gobrecht, J. and Müller, B., "Nano-mechanical transduction of polymer micro-cantilevers to detect bio-molecular interactions," Biointerfaces 7, 6 (2011).

[28] Schift, H., Park, S., Choi, C.-G., Kee, C.-S., Han, S.-P., Yoon, K.-B. and Gobrecht, J., "Fabrication process for polymer photonic crystals using nanoimprint lithography," Nanotechnol. 16, S261-S265 (2005).

[29] NaPa library of processes - nanopatterning and applications, Ed. H. Schift, published by the NaPa-consortium, $2^{\text {nd }}$ ed. (2012). <http://www.psi.ch/lmn/helmut-schift> (24 January 2014). http://www.psi.ch/lmn/helmut-schift

[30] Technology readiness level, <http://en.wikipedia.org/wiki/Technology_readiness_level> (24 January 2014). http://www.psi.ch/lmn/helmut-schift 